\title{
Biophysical mechanisms of single-cell interactions with microtopographical cues
}

\author{
Anuj A. Patel • Rahul G. Thakar • Matthew Chown • \\ Perla Ayala $\cdot$ Tejal A. Desai • Sanjay Kumar
}

Published online: 22 December 2009

(C) The Author(s) 2009. This article is published with open access at Springerlink.com

\begin{abstract}
Biophysical cues encoded in the extracellular matrix (ECM) are increasingly being explored to control cell behavior in tissue engineering applications. Recently, we showed that cell adhesion to microtopographical structures ("micropegs") can suppress proliferation in a manner that may be blunted by inhibiting cellular contractility, suggesting that this effect is related to altered cellscaffold mechanotransduction. We now directly investigate this possibility at the microscale through a combination of live-cell imaging, single-cell mechanics methods, and analysis of gene expression. Using time-lapse imaging, we show that when cells break adhesive contacts with micropegs, they form F-actin-filled tethers that extend and then
\end{abstract}

Electronic supplementary material The online version of this article (doi:10.1007/s10544-009-9384-7) contains supplementary material, which is available to authorized users.

A. A. Patel $\cdot$ M. Chown $\cdot$ S. Kumar $(\bowtie)$

Department of Bioengineering, University of California,

274A Stanley Hall \#1762,

Berkeley, CA 94720-1762, USA

e-mail: skumar@berkeley.edu

A. A. Patel $\cdot$ P. Ayala $\cdot$ T. A. Desai $\cdot$ S. Kumar

University of California San Francisco-University of California

Berkeley Joint Graduate Group in Bioengineering,

Berkeley, CA, USA

R. G. Thakar • P. Ayala • T. A. Desai

Department of Physiology, University of California,

203C Byers Hall Box 2520, 1700 4th St.,

San Francisco, CA 94158-2330, USA

R. G. Thakar • P. Ayala • T. A. Desai $(\square)$

Department of Bioengineering and Therapeutic Sciences,

University of California,

203C Byers Hall Box 2520, 1700 4th St.,

San Francisco, CA 94158-2330, USA

e-mail: tejal.desai@ucsf.edu rupture at a maximum, critical length that is greater than trailing-edge tethers observed on topographically flat substrates. This critical tether length depends on myosin activation, with inhibition of Rho-associated kinase abolishing topography-dependent differences in tether length. Using cellular de-adhesion and atomic force microscopy indentation measurements, we show that the micropegs enhance cell-scaffold adhesive interactions without changing whole-cell elasticity. Moreover, micropeg adhesion increases expression of specific mechanotransductive genes, including RhoA GTPase and myosin heavy chain II, and, in myoblasts, the functional marker connexin 43 . Together, our data support a model in which microtopographical cues alter the local mechanical microenvironment of cells by modulating adhesion and adhesion-dependent mechanotransductive signaling.

Keywords Microtopographical cues · Cell adhesion · Elasticity - AFM (atomic force microscopy) .

Tissue engineering

\section{Introduction}

One of the most important emerging themes in the field of bio-interfacial design is that biophysical signals encoded in biomaterial scaffolds may be used to control cell behavior independently of signals in the soluble milieu. For example, dictation of cell geometry by micropatterning the extracellular matrix (ECM) has been demonstrated to regulate cell proliferation and death (Chen et al. 1997; Nelson et al. 2005) and stem cell differentiation (McBeath et al. 2004; Ruiz and Chen 2008). Similarly, manipulation of ECM elasticity may be used to control a wide range of cellular functions, including adhesion and migration (Brown and 
Wong 2004; Peyton and Putnam 2005), lineage commitment and differentiation (Engler et al. 2006; Saha et al. 2008), and contractile and beating properties (Engler et al. 2008). Both shape- and rigidity-encoded cues are believed to be processed through mechanotransductive pathways that include activation and clustering of integrins, assembly and recruitment of focal adhesion proteins and cytoskeletal networks, and alterations in cellular mechanics and contractility (Chown and Kumar 2007; Kumar and Weaver 2009; Lele and Kumar 2007). In this sense, cells and the ECM participate in a reciprocal mechanical relationship in which cells adapt their intrinsic mechanical properties to ECM-encoded biophysical cues, as evidenced by the observations that changes in ECM stiffness and geometry can induce dramatic changes in cellular indentational elasticity (Sen et al. 2009; Solon et al. 2007).

While considerable attention has been paid to the role of ECM geometry and elasticity, much less is known about the contributions of scaffold topography. A variety of previous studies have shown that culturing cells on scaffolds containing 10-100 $\mu \mathrm{m}$-sized ridges and grooves promotes cell elongation and orientation (Clark et al. 1992; Miller et al. 2001; Recknor et al. 2004; Thakar et al. 2009), and this topographically-induced alignment has been used in combination with substrate stretch to promote vascular differentiation of mesenchymal stem cells (Kurpinski et al. 2006). Even scaffolds patterned with features much smaller than the size of the cell $(100 \mathrm{~nm}-1 \mu \mathrm{m})$ can strongly influence cell adhesion, assembly, and migration (Karuri et al. 2008; Liliensiek et al. 2006; Milner and Siedlecki 2007; Yim et al. 2005). Recent studies with corneal fibroblasts suggest that this effect may be due to altered nanoscale clustering of integrins, which in turn leads to altered focal adhesion assembly and cytoskeletal organization (Karuri et al. 2008). Similarly, culturing cells on scaffolds containing arrays of microscale protrusions (micropegs) promotes cell attachment and has the surprising effect of reducing cell proliferation (Boateng et al. 2003; Deutsch et al. 2000; Motlagh et al. 2003a, b). Even when micropegs are freed from their substrate and embedded in isotropic threedimensional gels, the structures serve as contact guidance cues for cells and support cell adhesion and elongation and cytoskeletal orientation, while still suppressing cell proliferation (Norman et al. 2008).

Seeking to understand mechanistic connections between ECM microtopography, cell proliferation, and mechanotransductive signaling, we recently cultured living fibroblasts and myoblasts on microfabricated scaffolds featuring arrays of micropegs (Thakar et al. 2008). Consistent with earlier observations, we found that adhesion to a micropeg substantially reduced the propensity of a cell to proliferate; this was accompanied by cell and nuclear elongation. Suspecting that micropeg adhesion might suppress proliferation through a contractility-dependent mechanism, we repeated these experiments in the setting of pharmacologic inhibition of Rho-associated kinase (ROCK) and myosin light-chain kinase (MLCK), indirect inhibitors of myosin, which strongly reduced the antiproliferative effects of micropeg adhesion. These results led us to speculate that micropeg adhesion might alter the mechanobiological properties of cells, including adhesive and migratory dynamics, mechanics, and expression of genes associated with mechanotransductive signaling. However, we did not directly test any of these hypotheses in our earlier report, leaving the micro- and nanoscale basis of cell-micropeg interactions an open question. Exploring these issues would lend mechanistic insight into the role of micropegs in controlling cellular assembly and proliferation in our system and, more generally, into the growing number of biomaterial and tissue engineering systems that seek to control cell behavior by encoding micro- and nanoscale topographical cues in the scaffold.

To address these challenges, we now directly demonstrate that micropeg adhesion alters the mechanobiological properties of living cells at the microscale. Through a combination of time-lapse phase and fluorescence imaging, cellular de-adhesion and atomic force microscopy (AFM) indentation measurements, and analysis of mechanotransductive gene expression, we show that adhesion to micropegs alters the local mechanical microenvironment by offering enhanced adhesive support to cells, which in turn enhances cell-scaffold mechanochemical feedback and amplifies expression of mechanotransductive genes.

\section{Materials and methods}

\subsection{Fabrication of PDMS micropegs}

PDMS micropeg arrays were fabricated as previously described (Thakar et al. 2008). Briefly, SU-8 2010 negative photoresist (PR) (Microchem, Newton, MA) was spincoated onto a single-crystal silicon wafer and baked at $95^{\circ} \mathrm{C}$ for $3 \mathrm{~min}$. Microscale holes were introduced by placing a patterned photomask over the coated wafer and exposing it to UV light for $25-30 \mathrm{~s}$ at an intensity of $5 \mathrm{~mW} \mathrm{~cm}^{-2}$, washing in SU-8 developer (Microchem) for $30 \mathrm{~s}$ to remove uncrosslinked PR, then baking at $95^{\circ} \mathrm{C}$ for $3 \mathrm{~min}$. To create PDMS micropeg arrays, PDMS and curing agent were prepared and mixed as directed by the manufacturer (Sylgard 184, Dow Corning, MI), degassed under vacuum, and spin-coated onto SU-8 mold. The PDMS was baked for $>2 \mathrm{~h}$ at $70^{\circ} \mathrm{C}$, then peeled from the SU-8 masters. Unpatterned PDMS membranes were fabricated in an identical manner, except for the use of non-PR-coated silicon wafers as masters. Prior to use in cell culture experiments, the PDMS was rendered hydrophilic by 
exposure to air or oxygen plasma and then incubated with mouse laminin (Invitrogen, Carlsbad, CA) at a concentration of $0.02 \mathrm{mg} \mathrm{mL}^{-1}$ in phosphate-buffered saline $(\mathrm{pH} 7.4)$ for $60 \mathrm{~min}$ at $4{ }^{\circ} \mathrm{C}$. For all studies, we used rectangular micropeg arrays featuring micropegs of $15 \mu \mathrm{m}$ height and $25 \mu \mathrm{m}$ diameter, and with nearest-neigbor array spacings of $50 \mu \mathrm{m}$ and $125 \mu \mathrm{m}$ center to center.

\subsection{Cell culture}

NIH 3 T3 mouse fibroblasts and $\mathrm{C} 2 \mathrm{C} 12$ mouse myoblasts (ATCC, Manassas, VA) were cultured on tissue culture plastic in complete medium consisting of Dulbecco's Modified Eagle's Medium (DMEM) (Gibco, Carlsbad, CA) with $10 \%$ calf serum (JR Scientific, Woodland, CA) and $1 \%$ penicillin/streptomycin (Gibco) for fibroblasts, and $10 \%$ fetal bovine serum (JR Scientific), 1\% sodium pyruvate (Gibco), and $1 \%$ penicillin/streptomycin for myoblasts. Cell cultures were stored in a humiditycontrolled $5 \% \mathrm{CO}_{2}$ incubator at $37^{\circ} \mathrm{C}$. For experiments, cells were allowed to grow to confluence, trypsinized, resuspended in complete medium, and plated on PDMS surfaces.

\subsection{Analysis of tether length}

To measure tether lengths, cells were plated on both flat and patterned PDMS substrates, allowed to spread for $4 \mathrm{~h}$, and then imaged for $20 \mathrm{~h}$. Tethers on each surface were measured at their maximum length before detaching or breaking. Approximately 50-60 tethers were measured per condition, and in cases in which a single cell sequentially formed and broke single tethers with one or more micropegs during timelapse imaging, each tether was measured and analyzed as a separate event. Where indicated, the MLCK inhibitor ML-7 or the ROCK inhibitor Y-27632 (Calbiochem, San Diego, CA) were diluted to $25 \mu \mathrm{M}$ in complete medium prior to addition to cultures. Cells were plated and allowed to spread for $4 \mathrm{~h}$ before addition of ML-7, then imaged for $20 \mathrm{~h}$.

\subsection{Live-cell fluorescence imaging}

Cells were plated on PDMS substrates and grown to 20$40 \%$ confluence in a $35 \mathrm{~mm}$ dish before transfection. The medium was then removed and replaced with $1 \mathrm{~mL}$ serum-free medium containing $10 \mu \mathrm{g}$ of Lipofectamine (Invitrogen) and $1.5 \mu \mathrm{g}$ of GFP-actin plasmid. Cells were incubated at $37^{\circ} \mathrm{C}$ for $24 \mathrm{~h}$, after which the medium was removed and replaced with complete medium. Images were taken with a Nikon TE2000E2 epifluorescence microscope.

\subsection{De-adhesion assay}

Trypsin de-adhesion was performed and analyzed as previously described (Sen and Kumar 2009). Briefly, cells plated on scaffolds were allowed to attach and spread for $24 \mathrm{~h}$. To assess de-adhesion, media was completely removed and replaced with warm $0.5 \%$ trypsin (Gibco). Images of cells were taken every $5 \mathrm{~s}$ until cells were rounded and no change in area could be observed. To quantify de-adhesion, cell area was measured by tracing the outline of the cell at various time points using ImageJ (NIH). The normalized area change over time was quantified by dividing the difference between the cell area at time $\mathrm{t}$ and the initial spread area $\left(A_{i}-A(t)\right)$ by the difference in area between the first and last time points $\left(A_{i}-A_{f}\right)$.

\subsection{Atomic force microscopy (AFM)}

Cells were cultured on PDMS as described above. Substrates were placed on a glass slide and mounted onto the stage of an Asylum MFP3D AFM (Asylum Research, CA) coupled to a Nikon TE2000E2 epifluorescence microscope. Cells were indented using a pyramid-tipped probe (Veeco Instruments, Santa Barbara, CA). Spring constants were determined using the thermal calibration method. Force curves were obtained for 30-35 cells for each condition. Each profile was fit with a modified Hertzian model of a cone indenting a semi-infinite elastic material to extract a set of elastic moduli.

\subsection{Western blotting}

Cells were allowed to adhere and spread on either flat or micropeg-patterned PDMS as above, followed by trypsinization and harvest of lysate. Protein levels were determined by Western blot, with detection by HRPconjugated secondary antibodies (Santa Cruz Biotechnology, Inc.) and development using Novex ECL chemiluminescent substrate (Invitrogen). ImageJ was used to determine band intensity levels from the developed blots. All intensity levels were internally normalized to the loading control ERK2 prior to calculating ratios of protein levels on micropeg-textured versus flat scaffolds. Note that lysate collected from flat scaffolds contains protein contributions only from cells adhered to a flat substrate, whereas lysate collected from micropeg-textured scaffolds contains the combined protein contributions of cells adhered to micropegs and cells adhered to the intervening flat regions. Thus, comparative analysis of lysates obtained from these two scaffold types yields a conservative underestimate of the effects of the micropegs on protein expression. 


\subsection{Statistics}

Results are presented as mean \pm standard error of the mean (SEM). Data are analyzed by Student's t-test for significance, except for Western blot analysis, which utilized a Log-transformed One-sample t-test due to the use of normalized values.

\section{Results}

3.1 Migrating cells form adhesive tethers on flat and micropeg-textured scaffolds

In our previous study, we cultured $3 \mathrm{~T} 3$ fibroblasts and C2 12 myoblasts on micropeg-textured, lamininfunctionalized PDMS scaffolds and showed that adhesion of either cell type to a micropeg produced a suite of phenotypic changes that included cell and nuclear elongation and reduced proliferation rate. These effects could be partially blocked by inhibiting ROCK or MLCK, which led us to postulate that the micropegs acted in part by altering cellular mechanobiological properties (Thakar et al. 2008). To gain additional insight into adhesive interactions between an individual cell and a micropeg, we began here by using phase-contrast imaging to obtain time-lapse movies of 3T3 fibroblasts randomly migrating on the same flat and micropeg-textured PDMS scaffolds we had used previously (Fig. 1; Supplementary Movies 1 2, 3, 4, 5 and 6). Cells migrating on flat substrates developed welldefined leading and trailing edges, with the trailing edge forming "tethers" that thinned, extended, and eventually ruptured as the cell body translocated forward (Fig. 1(A), Supplementary Movie 1). We continued to observe these tethers when we pharmacologically inhibited either MLCK (Fig. 1(B), Supplementary Movie 2) or ROCK (Fig. 1(C), Supplementary Movie 3) under conditions we had shown earlier were capable of blocking the effects of micropegs on proliferation. When we repeated these experiments on micropeg-textured substrates (Figs. 1(D-F), Supplementary Movies 4, 5 and 6), we noticed that fibroblasts migrating away from micropegs formed similar adhesive tethers with the micropegs as they broke adhesive contact, with the tethers also persisting in the setting of either MLCK or ROCK inhibition.

3.2 Tether length is modulated by scaffold microtopography and cell contractility

Retraction of the trailing edge of cells during migration reflects a mechanical balance between cell-generated contractile forces and cell-scaffold adhesive contacts (Palecek et al. 1998). Thus, we reasoned that the length at which these tethers rupture (i.e., the maximum tether length) might reflect the relative strength of cellular contractility and cell-scaffold adhesion and therefore change with scaffold microtopography and the activation of myosin-based contractility. To verify that the tethers observed in our system contain contractile elements and are not empty membrane tethers, we transfected fibroblasts with green fluorescent protein-tagged actin (GFP-actin) and used time-lapse epifluorescence imaging to image actin cytoskeletal dynamics during tether retraction (Fig. 2(A), Supplementary Movie 7). Indeed, we found that the tethers contained actin-positive bundles that were contiguous with the cell's peripheral stress fiber network.

We next performed quantitative morphometric analysis to extract average maximum tether lengths in for cells cultured on flat and micropeg-textured scaffolds, and in the presence and absence of MLCK and ROCK inhibition (Fig. 2B). We observed that in the absence of inhibitor, cells cultured on micropeg-textured scaffolds exhibited greater mean tether lengths than cells cultured on flat scaffolds, suggesting that the micropegs alter cell-micropeg adhesive interactions, contractile interactions, or both. This difference persisted in the setting of MLCK inhibition; remarkably, the average maximum tether length on micropeg-textured scaffolds under MLCK inhibition was comparable to that observed on flat scaffolds without drug, suggesting that adhesion to a micropeg might rescue the effects of MLCK inhibition. Interestingly, ROCK inhibition abolished tether length differences between flat and micropeg-textured scaffolds and increased tether lengths overall, consistent with previous studies with other cell types (Ulrich et al. 2009) and reflecting reduced contractile forces at the trailing edge.

\subsection{Cell de-adhesion is slowed by attachment to a micropeg}

To gain additional quantitative insight into how micropeg engagement might regulate cell-scaffold adhesion and mechanics, we applied a modified version of a trypsin deadhesion assay we recently developed, in which we enzymatically detach adherent cells and quantify the rate at which the cells round, expressed as the change in normalized projected cell area as a function of time (see Methods) (Sen and Kumar 2009). When we conducted this assay for cells cultured on flat and micropeg-textured scaffolds (Fig. 3, Supplemental Movies 8 and 9), we observed that cells in contact with a micropeg detached from the surface more slowly than cells on a flat scaffold, indicating that the micropegs either decrease cell contractility or increase adhesion strength. The notion that the micropegs might enhance scaffold adhesion is further supported by the observation that on micropeg-textured scaffolds, which contain both micropegs and intervening 

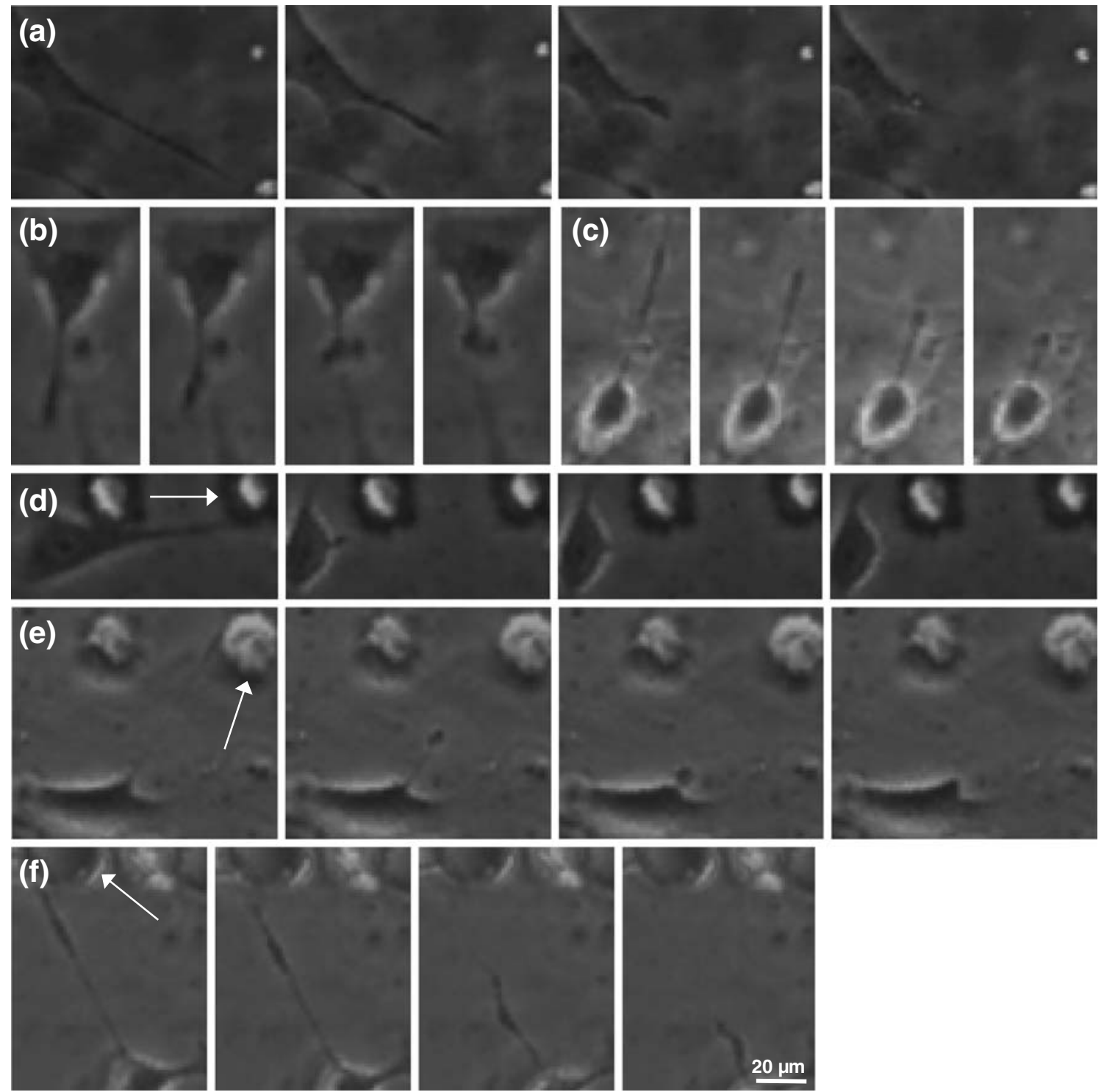

Fig. 1 Adhesive tether rupture and retraction on flat and micropegtextured scaffolds. 3T3 fibroblasts were cultured on flat (A-C) or micropeg-textured (D-F, note white arrows) laminin-coated PDMS scaffolds and followed by phase-contrast time-lapse imaging in the

flat regions, cells associated with a micropeg remained attached to the scaffold much longer than their counterparts on flat regions of the scaffold during enzymatic digestion (data not shown).

\subsection{Micropeg adhesion does not significantly alter whole-cell elasticity}

Taken together, our tether length analysis and de-adhesion assays indicate that micropeg attachment alters either cellular adhesion, contractile mechanics, or both. To measure contributions of micropeg adhesion to whole-cell contractile mechanics in a more isolated fashion, we used presence of no drug (A, D), ML-7 (B, E), or Y-27632 (C, F). In all cases, the elapsed time between adjacent frames is $1 \mathrm{~min}$. Scale bar $=20 \mu \mathrm{m}$

AFM indentation to measure the cortical elasticity of cells on flat and micropeg-textured surfaces (Fig. 4). Cells cultured on flat and micropeg-textured substrates had mean elasticities of 3.92 and $3.05 \mathrm{kPa}$, respectively, a range consistent with our and others' previous measurements across multiple cell types (Sen and Kumar 2009; Solon et al. 2007). Although cells cultured on flat substrates "trend" toward higher stiffnesses than those attached to micropegs, the difference is both statistically insignificant and small compared to the $>5$-fold differences in cell stiffnesses reported to be induced by pharmacologic dissipation of actomyosin contractility (Rotsch et al. 1997). Thus, micropeg adhesion at best minimally alters global cellular 
Fig. 2 Adhesive tethers contain F-actin bundles and change with MLCK and ROCK inhibition. (A) Time-lapse imaging of GFP-actin. 3T3 fibroblasts were transfected with GFP-actin and imaged for $5 \mathrm{~h}$ to capture adhesive interactions with the scaffold. 3T3 fibroblast tether retraction is shown at initial tether, tether immediately before rupture, and immediately after retraction. Cells were transfected with GFP-actin and imaged for $5 \mathrm{~h}$. Tethers feature prominent F-actin-based bundles that appear contiguous with the cellular stress fiber network and retract into the cell body over time. (B) Maximum tether lengths in the presence and absence of topographical cues and the contractility inhibitors ML-7 and Y-27632 $(* p<0.05$ by ANOVA followed by t-test). Error bars represent standard errors of the mean (a)

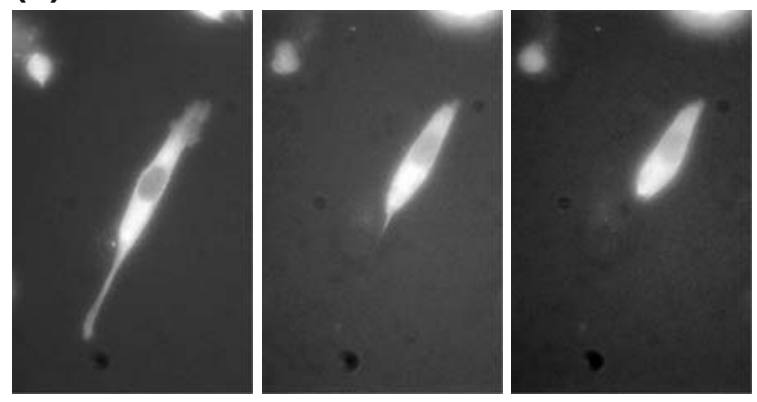

(b)

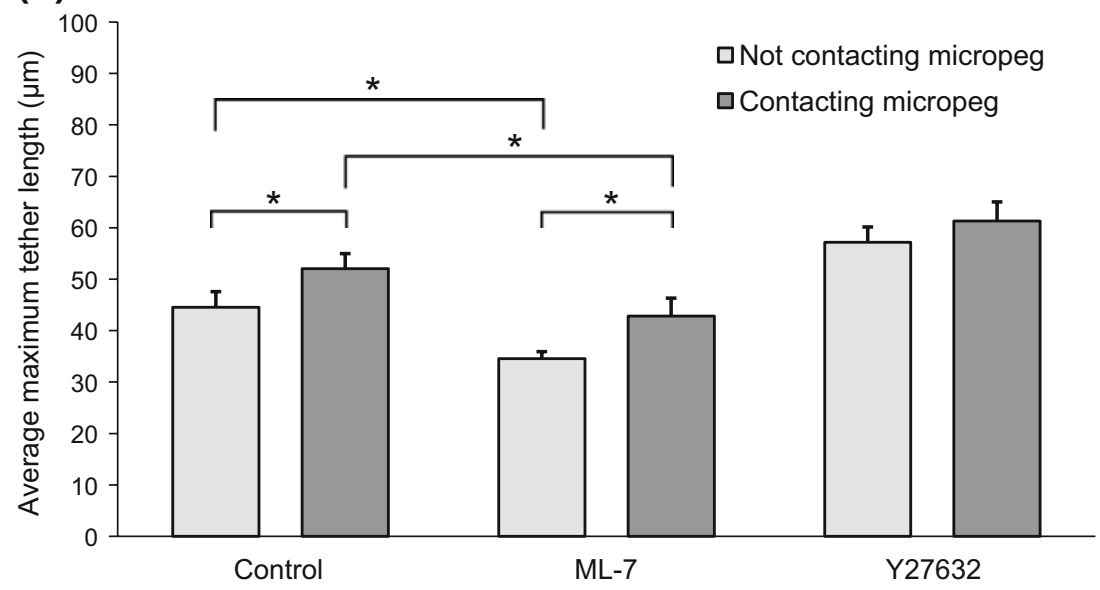

contractile mechanics, which strongly suggests that the changes in tether and de-adhesion dynamics are primarily due to changes in cell-scaffold adhesion.

3.5 Micropeg adhesion alters expression of Myosin Heavy Chain, RhoA GTPase, and Connexin 43

While our AFM measurements failed to demonstrate significant regulation by micropegs of whole-cell contractile mechanics, it occurred to us that micropeg adhesion might still alter the expression of genes relevant to cell-scaffold mechanobiology, including elements of the myosin contractility pathway. To explore this possibility, we used Western blotting to compare the expression of selected proteins on flat versus micropeg-textured scaffolds (Fig. 5). C2C12 cells attached to micropegs exhibited increased expression of both RhoA GTPase and myosin heavy chain II (MYH2), which are critical to the assembly and activity of contractile stress fiber bundles. Importantly, because protein lysates obtained from micropeg-textured scaffolds are partially "diluted" by the contributions of cells adherent to flat regions that lie between the micropegs, any observed differences in protein expression between flat and micropeg-textured scaffolds provide a conservative underestimate of micropeg-induced effects. Interestingly, myoblasts on micropeg-textured scaffolds also displayed enhanced expression of Connexin43 (Cxn43), which plays a critical role in the fusion and differentiation of individual myoblasts into multicellular myotubes (Araya et al. 2003; Squecco et al. 2006), hinting that micropeg adhesion may promote myoblast maturation.

\section{Discussion}

In this study, we have used live-cell time-lapse imaging, AFM, and analysis of protein expression to investigate the role played by microtopographical cues on the adhesion and mechanics of single cells. Our data support a model in which these cues alter the local physical microenvironment of cultured cells by enhancing adhesive interactions and expression of proteins that participate in cell-scaffold mechanochemical feedback. This also builds directly on our previous study, which showed that adhesion to a micropeg suppresses proliferation in a manner that can be blocked by inhibiting the ability of the cell to generate force against the scaffold, suggesting that the micropegs control cell behavior in part by altering cell-scaffold biomechanical feedback. Our current results provide the first direct evidentiary support for that hypothesis by directly demonstrating that the micropegs alter these 

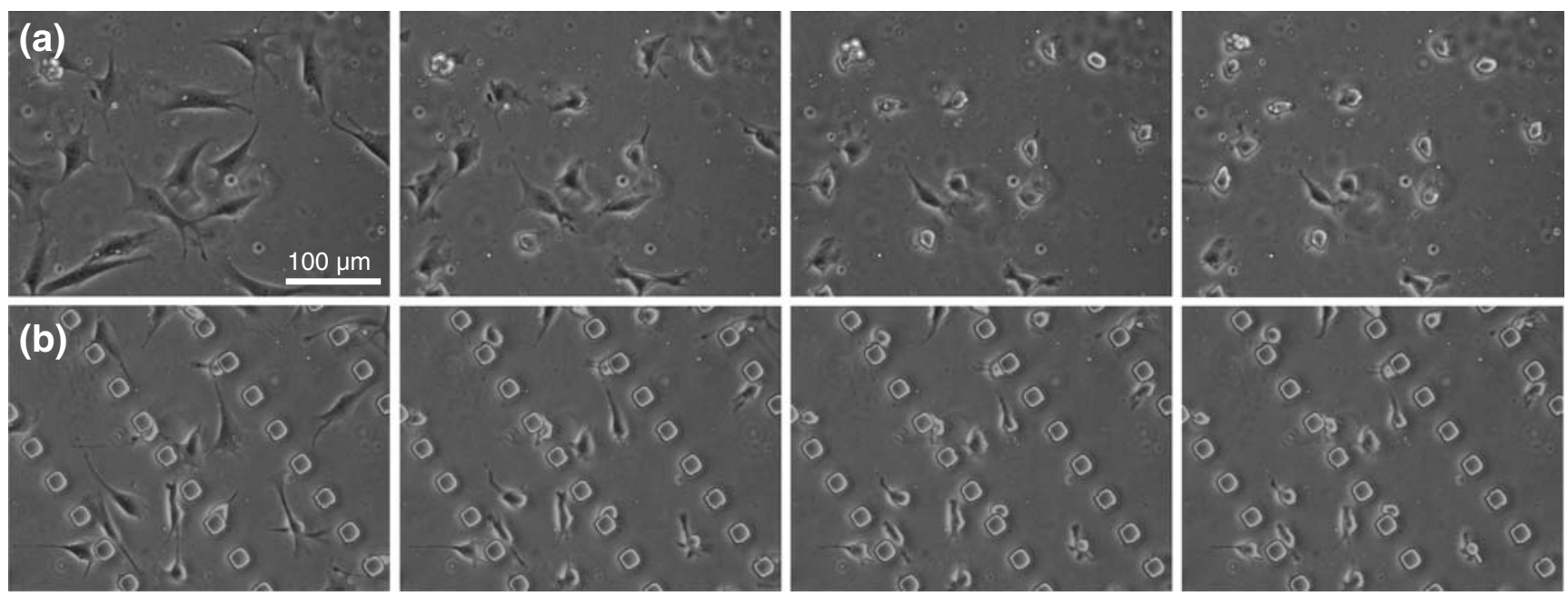

(c)

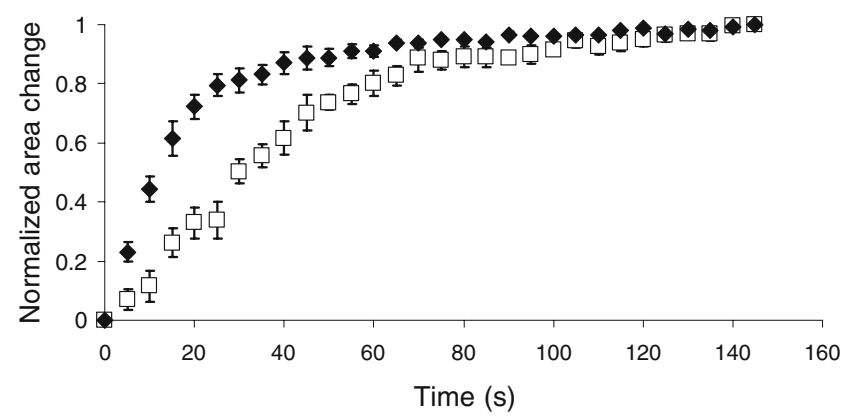

Fig. 3 Micropegs alter de-adhesive dynamics. (A)-(B) Time-lapse imaging of trypsin-induced de-adhesion and retraction of $\mathrm{C} 2 \mathrm{C} 12$ skeletal myoblasts on (A) flat and (B) micropeg-textured scaffolds. Cells were plated on each surface, allowed to spread for $24 \mathrm{~h}$, and then detached with trypsin as previously described [29]. The time elapsed

between each panel is $1 \mathrm{~min}$. (C) Normalized area change vs. time on flat (diamonds) and micropeg-textured (squares) scaffolds. Normalized area change is defined as $\left[A_{i}-A(t)\right] /\left[A_{i}-A_{f}\right]$ where $A_{i}$ is the initial area, $A(t)$ is the area at time $t$, and $A_{f}$ is the final area. Error bars represent standard errors of the mean

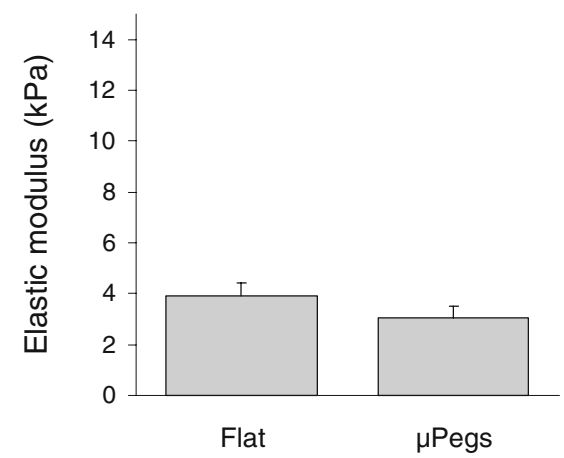

Fig. 4 Micropegs do not significantly alter cell stiffness. AFM indentation of cells indicates that the elastic modulus between cells attached to micropegs and cells on flat surface are not significantly different. Cells were seeded on surfaces and allowed to attach for $24 \mathrm{~h}$ before taking measurements. Measurements of cells attached to micropegs are taken as close to the micropeg as possible. Each condition represents $30-40$ cells being indented with $p>0.1$ by standard t-Test. Error bars indicate SEM

biophysical interactions; to our knowledge, this is also the first detailed microscale analysis of mechanical and adhesive interactions between cells and microtopographical features.

Our initial hint that engagement of a micropeg might alter cell-scaffold adhesion came from observations of tethers formed by the trailing edges of cells as they migrate away and break adhesive contact from micropegs. Extrusion and characterization of membrane tethers has been used on many previous occasions to quantify local membrane and cytoskeletal mechanics, typically in the context of optical or magnetic tweezer studies (Girdhar and Shao 2007; Tabdanov et al. 2009; Titushkin and Cho 2006, 2007). In these studies, membrane tethers are much smaller $(<1 \mu \mathrm{m})$ than those observed here and devoid of cytoskeletal components. However, even under those circumstances, critical tether lengths are highly sensitive to both the state of the cytoskeleton and particle-tether adhesivity. For example, Titushkin and Cho found 2.5-fold increases in maximum tether length following cytochalasin-mediated disruption of the actin cytoskeleton in osteoblasts, 

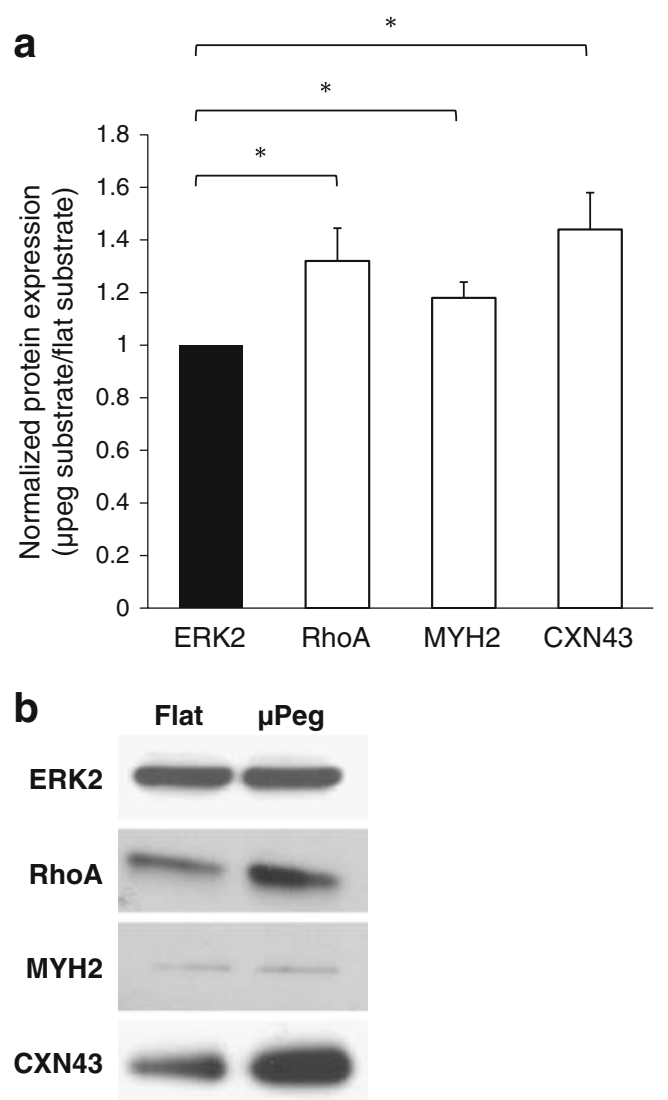

Fig. 5 Effect of micropegs on expression of selected mechanobiological genes. (A) Calculation of ratio of protein expression on micropegtextured scaffolds to flat scaffolds, as measured by Western Blot. Both expression levels were normalized to expression of the loading control ERK2 on each scaffold. Expression of RhoA GTPase (RhoA), myosin heavy chain II (MYH2), and connexin 43 (CXN43) was statistically significantly higher on micropeg-textured scaffolds than on flat scaffolds $\left({ }^{*} p<0.05\right.$ by log-transformed one-sample t-test). Error bars indicate SEM over at least 3 independent experiments. (B) Representative raw Western blot data for ERK2, RhoA, MYH2, and CXN43

corresponding to a $47 \%$ reduction in cortical elasticity by AFM (Titushkin and Cho 2007).

In migrating cells, retraction of the trailing edge has been widely described as reflecting a balance between cellgenerated contractile forces and cell-scaffold adhesion, with enhanced adhesion to the substrate and reduced cytoskeletal contractility promoting greater tether lengths (Palecek et al. 1998, 1996; Rid et al. 2005; Ulrich et al. 2009). Indeed, maximum process length has been used previously as a semi-quantitative metric of this force balance; for example, Iwanicki and colleagues recently reported that serumstarved fibroblasts form trailing-edge tethers that are approximately fourfold longer than those observed in serum-cultured controls and that this difference can be eliminated by lysophosphatidic acid-mediated stimulation of RhoA GTPase, which would be expected to potentiate contractility. Conversely, Palecek and colleagues observed that highly adhesive scaffolds slow cell migration by limiting the rate of trailing-edge detachment, which is in turn accompanied by production of broad and highly stable lamellipodia (Palecek et al. 1997). Placing our findings in the context of this paradigm, we hypothesize that the micropegs provide an enhanced adhesive environment that supports greater tether lengths than flat scaffolds; whether this results from the provision of additional integrin ligation sites, a locally three-dimensional environment, or some combination of the two remains unclear.

While we do not find substantial micropeg-induced changes in cortical mechanics (Fig. 4), we do observe significant upregulation of expression of several elements of the myosin contractility pathway, including Rho GTPase and myosin II heavy chain (Fig. 5). An interesting explanation for these data is that micropeg adhesion induces only local changes in cellular mechanobiology, without altering the mechanics of the entire cell. Under this scenario, micropeg adhesion might enhance adhesiondependent signaling and assembly of contractile structures only in the immediate vicinity of the micropeg without altering global cell mechanics. Here, contractility proteins or their mRNA precursors might be preferentially trafficked towards these new adhesive/contractile structures, analogous to previous observations of ribosomal recruitment to newly-formed focal adhesions (Chicurel et al. 1998).

Our study adds new intracellular, microscale insight into a rich body of evidence that illustrates how powerfully micro- and nanoscale topographical cues can regulate cell adhesion and adhesion-dependent signaling in a manner that is largely independent of soluble biochemical cues (Kurpinski et al. 2006; Motlagh et al. 2003a; Recknor et al. 2004). Intriguingly, these cues can profoundly influence cell behavior even when the textured structures are orders of magnitude smaller than the cells themselves. For example, culturing smooth muscle cells on substrates nanopatterned with grooves of only $350 \mathrm{~nm}$ in width causes alignment along the grooves and suppression of cell proliferation (Yim et al. 2005). Similarly, the proliferation of corneal fibroblasts can be modulated over a wide range through incorporation of nanoscale ridges and grooves into the scaffold, with smaller pitch sizes suppressing proliferation the most (Liliensiek et al. 2006). Even non-oriented micro- and nanoscale topographical features can strongly regulate cell behavior; for example, nonspecific decoration of planar scaffolds with metal oxide-based nanorods restricts cell spreading, and deposition of these nanorods into spatially-defined positions on the scaffold can be used as the basis of cell patterning (Lee et al. 2009, 2008). In virtually all of these cases, the nanotextured structures have been proposed to act by regulating integrin clustering and focal adhesion at the nanoscale, thereby influencing all downstream adhesion-dependent signaling. In our case, the 
microtextured structures are on the order of micrometers, i.e., larger than the features considered in these studies but on the same size scale as a single focal adhesion. We speculate that the enhanced adhesion and altered adhesiondependent signaling that we observe may be derived from altered assembly of adhesive structures at the cell-micropeg interface, and we anticipate that by labeling and imaging adhesive structures at very high spatial and temporal resolution, we may be able to explore this possibility in greater detail.

\section{Conclusions}

We have explored biophysical interactions between single cells and microtopographical protrusions ("micropegs"). Using live-cell phase-contrast and fluorescence imaging, single-cell de-adhesion measurements, and AFM, we have shown that micropeg adhesion enhances cell-scaffold adhesion without altering global cell mechanics. Comparative analysis of gene expression reveals that micropeg adhesion also amplifies expression of the mechanotransductive proteins RhoA GTPase and myosin heavy chain II. We hypothesize that micropeg engagement locally reinforces cell-scaffold adhesive contacts, which in turn modulates adhesion-dependent signaling and may account for the ability of the micropegs to suppress cell proliferation.

Acknowledgments This work was supported by a Beginning Grant-in-Aid from the American Heart Association (0765128Y to S.K.), a Beckman Young Investigator Award (to S.K.), and the NIH Director's New Innvoator Award, part of the the NIH Roadmap for Medical Research (to S. K.). It was also supported by the Sandler Family Foundation (to T.A.D) and the NSF (NSEC to T.A.D. and GSRP to P.A.). We would also like to thank the Nikon Imaging Center and the Biomedical Micro/Nanotechnology Core at UCSF.

Open Access This article is distributed under the terms of the Creative Commons Attribution Noncommercial License which permits any noncommercial use, distribution, and reproduction in any medium, provided the original author(s) and source are credited.

\section{References}

R. Araya, D. Eckardt, M.A. Riquelme, K. Willecke, J.C. Saez, Presence and importance of connexin 43 during myogenesis. Cell Commun. Adhes. 10, 451-456 (2003)

S.Y. Boateng, T.J. Hartman, N. Ahluwalia, H. Vidula, T.A. Desai, B. Russell, Inhibition of fibroblast proliferation in cardiac myocyte cultures by surface microtopography. Am. J. Physiol. Cell Physiol. 285, C171-C182 (2003)

X.Q. Brown, J.Y. Wong, Substrate stiffness enhances the stimulatory effect of PDGF on VSMC. Mol. Biol. Cell 15, 301A-301A (2004)

C.S. Chen, M. Mrksich, S. Huang, G.M. Whitesides, D.E. Ingber, Geometric control of cell life and death. Science 276, 1425-1428 (1997)
M.E. Chicurel, R.H. Singer, C.J. Meyer, D.E. Ingber, Integrin binding and mechanical tension induce movement of mRNA and ribosomes to focal adhesions. Nature 392, 730-733 (1998)

M.G. Chown, S. Kumar, Imaging and manipulating the structural machinery of living cells on the micro- and nanoscale. Int. J. Nanomedicine 2, 333-344 (2007)

P. Clark, P. Connolly, G.R. Moores, Cell guidance by micropatterned adhesiveness invitro. J. Cell Sci. 103, 287-292 (1992)

J. Deutsch, D. Motlagh, B. Russell, T.A. Desai, Fabrication of microtextured membranes for cardiac myocyte attachment and orientation. J. Biomed. Mater Res. 53, 267-275 (2000)

A.J. Engler, C. Carag-Krieger, C.P. Johnson, M. Raab, H.Y. Tang, D.W. Speicher, J.W. Sanger, J.M. Sanger, D.E. Discher, Embryonic cardiomyocytes beat best on a matrix with heart-like elasticity: scar-like rigidity inhibits beating. J. Cell Sci. 121, 3794-3802 (2008)

A.J. Engler, S. Sen, H.L. Sweeney, D.E. Discher, Matrix elasticity directs stem cell lineage specification. Cell 126, 677-689 (2006)

G. Girdhar, J.Y. Shao, Simultaneous tether extraction from endothelial cells and leukocytes: observation, mechanics, and significance. Biophys. J. 93, 4041-4052 (2007)

N.W. Karuri, P.F. Nealey, C.J. Murphy, R.M. Albrecht, Structural organization of the cytoskeleton in SV40 human corneal epithelial cells cultured on nano- and microscale grooves. Scanning 30, 405413 (2008)

S. Kumar, V. Weaver, Mechanics, malignancy, and metastasis: the force journey of a tumor cell. Cancer Metastasis Rev. 28, 113127 (2009)

K. Kurpinski, J. Chu, C. Hashi, and S. Li, Anisotropic mechanosensing by mesenchymal stem cells. Proceedings of the National Academy of Sciences of the United States of America. 103, 16095-16100 (2006)

J. Lee, B.H. Chu, K.-H. Chen, F. Ren, T.P. Lele, Randomly oriented, upright $\mathrm{SiO} 2$ coated nanorods for reduced adhesion of mammalian cells. Biomaterials 30, 4488-4493 (2009)

J.Y. Lee, B.S. Kang, B. Hicks, T.F. Chancellor, B.H. Chu, H.T. Wang, B.G. Keselowsky, F. Ren, T.P. Lele, The control of cell adhesion and viability by zinc oxide nanorods. Biomaterials 29, 3743 3749 (2008)

T.P. Lele, S. Kumar, Brushes, cables, and anchors: recent insights into multiscale assembly and mechanics of cellular structural networks. Cell Biochem. Biophys. 47, 348-360 (2007)

S.J. Liliensiek, S. Campbell, P.F. Nealey, C.J. Murphy, The scale of substratum topographic features modulates proliferation of corneal epithelial cells and corneal fibroblasts. J. Biomed. Mater. Res. A 79A, 185-192 (2006)

R. McBeath, D.M. Pirone, C.M. Nelson, K. Bhadriraju, C.S. Chen, Cell shape, cytoskeletal tension, and RhoA regulate stem cell lineage commitment. Developmental Cell 6, 483-495 (2004)

C. Miller, H. Shanks, A. Witt, G. Rutkowski, S. Mallapragada, Oriented Schwann cell growth on micropatterned biodegradable polymer substrates. Biomaterials 22, 1263-1269 (2001)

K.R. Milner, C.A. Siedlecki, Fibroblast response is enhanced by poly (L-lactic acid) nanotopography edge density and proximity. Int. J. Nanomedicine 2, 201-211 (2007)

D. Motlagh, T.J. Hartman, T.A. Desai, B. Russell, Microfabricated grooves recapitulate neonatal myocyte connexin 43 and $\mathrm{N}$ cadherin expression and localization. J. Biomed. Mater. Res. A 67, 148-157 (2003a)

D. Motlagh, S.E. Senyo, T.A. Desai, B. Russell, Microtextured substrata alter gene expression, protein localization and the shape of cardiac myocytes. Biomaterials 24, 2463-2476 (2003b)

C.M. Nelson, R.P. Jean, J.L. Tan, W.F. Liu, N.J. Sniadecki, A.A. Spector, and C.S. Chen, Emergent patterns of growth controlled by multicellular form and mechanics. Proceedings of the National Academy of Sciences of the United States of America 102, 11594-11599 (2005). 
J.J. Norman, J.M. Collins, S. Sharma, B. Russell, T.A. Desai, Microstructures in 3D biological gels affect cell proliferation. Tissue Engineering Part A 14, 379-390 (2008)

S.P. Palecek, A. Huttenlocher, A.F. Horwitz, D.A. Lauffenburger, Physical and biochemical regulation of integrin release during rear detachment of migrating cells. J. Cell Sci. 111, 929-940 (1998)

S.P. Palecek, J.C. Loftus, M.H. Ginsberg, D.A. Lauffenburger, A.F. Horwitz, Integrin-ligand binding properties govern cell migration speed through cell-substratum adhesiveness. Nature 385, 537540 (1997)

S.P. Palecek, C.E. Schmidt, D.A. Lauffenburger, A.F. Horwitz, Integrin dynamics on the tail region of migrating fibroblasts. J. Cell Sci. 109, 941-952 (1996)

S.R. Peyton, A.J. Putnam, Extracellular matrix rigidity governs smooth muscle cell motility in a biphasic fashion. J. Cell Physiol. 204, 198-209 (2005)

J.B. Recknor, J.C. Recknor, D.S. Sakaguchi, S.K. Mallapragadaa, Oriented astroglial cell growth on micropatterned polystyrene substrates. Biomaterials 25, 2753-2767 (2004)

R. Rid, N. Schiefermeier, I. Grigoriev, J.V. Small, I. Kaverina, The last but not the least: the origin and significance of trailing adhesions in fibroblastic cells. Cell Motil. Cytoskelet. 61, 161171 (2005)

C. Rotsch, F. Braet, E. Wisse, M. Radmacher, AFM imaging and elasticity measurements on living rat liver macrophages. Cell Biol. Int. 21, 685-696 (1997)

S.A. Ruiz, C.S. Chen, Emergence of patterned stem cell differentiation within multicellular structures. Stem Cells 26, 2921-2927 (2008)

K. Saha, A.J. Keung, E.F. Irwin, Y. Li, L. Little, D.V. Schaffer, K.E. Healy, Substrate modulus directs neural stem cell behavior. Biophys. J. 95, 4426-4438 (2008)

S. Sen, S. Kumar, Cell-matrix de-adhesion dynamics reflect contractile mechanics. Cell Mol. Bioeng. 2, 218-230 (2009)
S. Sen, M. Dong, S. Kumar, Isoform-specfic contributions of aactinin to glioma cell mechanobiology. PLOS ONE (http://dx. plos.org/10.1371/journal.pone.0008427) (2009)

J. Solon, I. Levental, K. Sengupta, P.C. Georges, P.A. Janmey, Fibroblast adaptation and stiffness matching to soft elastic substrates. Biophys. J. 93, 4453-4461 (2007)

R. Squecco, C. Sassoli, F. Nuti, M. Martinesi, F. Chellini, D. Nosi, S. Zecchi-Orlandini, F. Francini, L. Formigli, E. Meacci, Sphingosine 1-phosphate induces myoblast differentiation through Cx43 protein expression: a role for a gap junction-dependent and independent function. Mol. Biol. Cell 17, 4896-4910 (2006)

E. Tabdanov, N. Borghi, F. Brochard-Wyart, S. Dufour, J.P. Thiery, Role of e-cadherin in membrane-cortex interaction probed by nanotube extrusion. Biophys. J. 96, 2457-2465 (2009)

R.G. Thakar, Q. Cheng, S. Patel, J. Chu, M. Nasir, D. Liepmann, K. Komvopoulos, S. Li, Cell-shape regulation of smooth muscle cell proliferation. Biophys. J. 96, 3423-3432 (2009)

R.G. Thakar, M.G. Chown, A. Patel, L. Peng, S. Kumar, T.A. Desai, Contractility-dependent modulation of cell proliferation and adhesion by microscale topographical cues. Small 4, 1416-1424 (2008)

I. Titushkin, M. Cho, Distinct membrane mechanical properties of human mesenchymal stem cells determined using laser optical tweezers. Biophys. J. 90, 2582-2591 (2006)

I. Titushkin, M. Cho, Modulation of cellular mechanics during osteogenic differentiation of human mesenchymal stem cells. Biophys. J. 93, 3693-3702 (2007)

T.A. Ulrich, E.M. de Juan Pardo, S. Kumar, The mechanical rigidity of the extracellular matrix regulates the structure, motility, and proliferation of glioma cells. Cancer Res. 69, 4167-4174 (2009)

E.K.F. Yim, R.M. Reano, S.W. Pang, A.F. Yee, C.S. Chen, K.W. Leong, Nanopattern-induced changes in morphology and motility of smooth muscle cells. Biomaterials 26, 5405-5413 (2005) 